\title{
Heart transplantation for Behcet's disease after three aortic root replacements
}

\author{
Hyo-Hyun Kim^, Young-Ho Yang, Seung Hyun Lee^, Young-Nam Youn^^ \\ Division of Cardiovascular Surgery, Severance Cardiovascular Hospital, Yonsei University College of Medicine, Yonsei University Health System, \\ Seoul, Republic of Korea \\ Correspondence to: Young-Nam Youn, MD, PhD. Division of Cardiovascular Surgery, Severance Cardiovascular Hospital, Yonsei University College of \\ Medicine, Yonsei University Health System, 250 Seongsanno, Seodaemun-gu, Seoul 03722, Korea. Email: ynyoun@yuhs.ac. \\ Provenance and Peer Review: This article was a free submission to the journal, fournal of Thoracic Disease. The article did not undergo external peer review.
}

Submitted Feb 29, 2020. Accepted for publication May 28, 2020.

doi: 10.21037/jtd-20-1084

View this article at: http://dx.doi.org/10.21037/jtd-20-1084

\section{Introduction}

Behcet's disease (BD) is an autoimmune disorder involving the gastrointestinal, cardiovascular, and central nervous systems, characterized by relapsing uveitis and recurrent oral and genital ulcers (1). Reports of surgical treatment in patients with $\mathrm{BD}$ are scarce and despite the necessary treatments, the long-term survival rate is low (2). Moreover, cardiac transplantation is deemed a high-risk procedure (3). We report a case of heart transplantation performed after the patient underwent three aortic root replacement procedures due to $\mathrm{BD}$.

\section{Case presentation}

In 2016, a 49-year-old Asian man presented with recurrent oral ulceration, pustular response to the skin, and dyspnea (New York Heart Association class III). Markers of inflammation, such as C-reactive protein and erythrocyte sedimentation rate, were elevated $(68.9 \mathrm{mg} / \mathrm{L}$ and $22 \mathrm{~mm} / \mathrm{h}$, respectively). In addition, we suspected aortic regurgitation (AR) and diastolic mitral regurgitation as cardiovascular manifestations of BD. After being on medical treatment with prednisolone (Solondo, Yuhan. Corp., Seoul, Korea, $20 \mathrm{mg} /$ day) for 2 years, the patient presented again with chief complaints of sudden onset chest and back pain. Coronary angiography revealed no coronary artery stenosis, and cardiac enzymes were within normal limits. However, cardiac imaging tests revealed a dilated ascending aorta $(55 \mathrm{~mm})$, suspicious dissection, and an unusual aortic wall thickening suggestive of aortitis. Despite several preoperative medications, the worsening of $\mathrm{AR}$ and sinus of Valsalva aneurysm were observed (Video 1).

During the first procedure, the aortic wall was severely thickened with chronic dissection that extended to the lesser curvature of the aortic arch. Valsalva aneurysm and non-coronary cusp perforation were observed (panel A in Figure 1). Therefore, the patient underwent an annular reconstruction using bovine pericardium, an aortic root replacement procedure with mechanical valved conduit, and a graft replacement of the hemiarch. At discharge, maintenance therapy with methotrexate (Yuhan. Corp., Seoul, Korea, $10 \mathrm{mg} /$ week $)$ and prednisolone $(60 \mathrm{mg} /$ day $)$ was prescribed. Almost 4 months later, inflammatory markers were increased again and multiple pseudoaneurysms extending to sub- and supra-valvular areas were detected on transesophageal echocardiography and cardiac computed tomography (panel A in Figure 2). To prevent the rupture of the aortic root, he underwent a second aortic root replacement using homograft.

After 5 months, the patient developed a high fever and his inflammation relapsed. Despite administering high-dose intravenous steroid pulse therapy, there were newly formed

\footnotetext{
^ Hyo-Hyun Kim, ORCID: 0000-0002-1608-9674; Seung Hyun Lee, ORCID: 0000-0002-0311-6565; Young-Nam Youn, ORCID: 0000$0002-7755-1877$.
} 

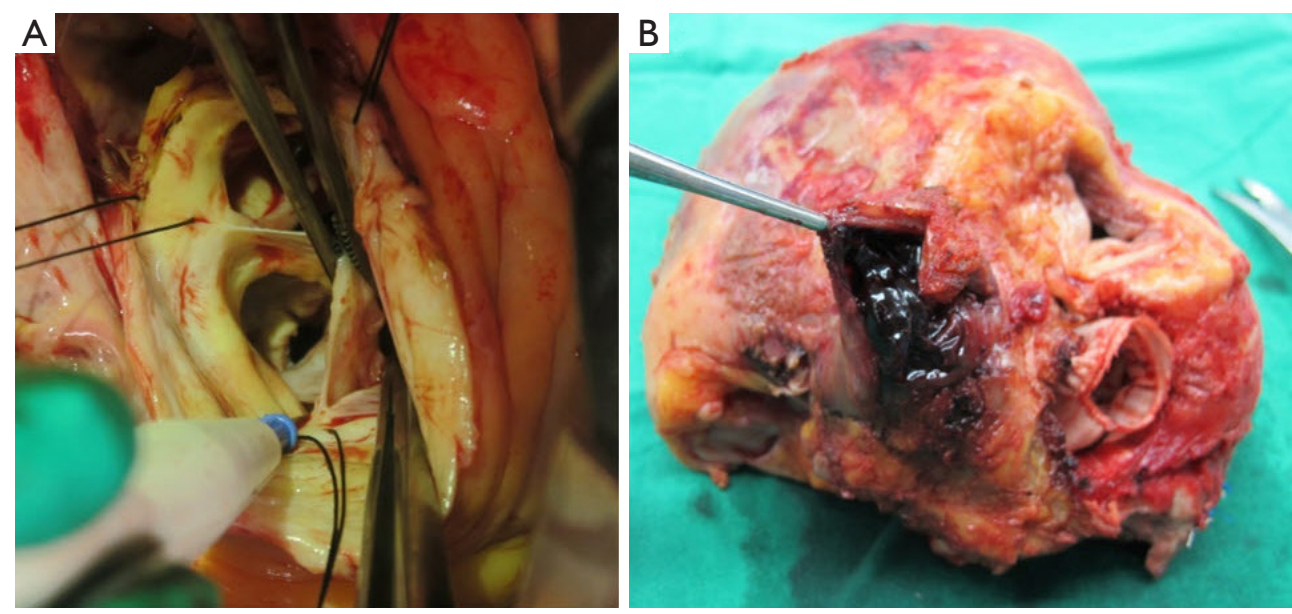

Figure 1 Operative findings. (A) Dissection flap and non-coronary cusp perforation; (B) aneurysmal change of the left atrial wall and thrombus inside the sac.
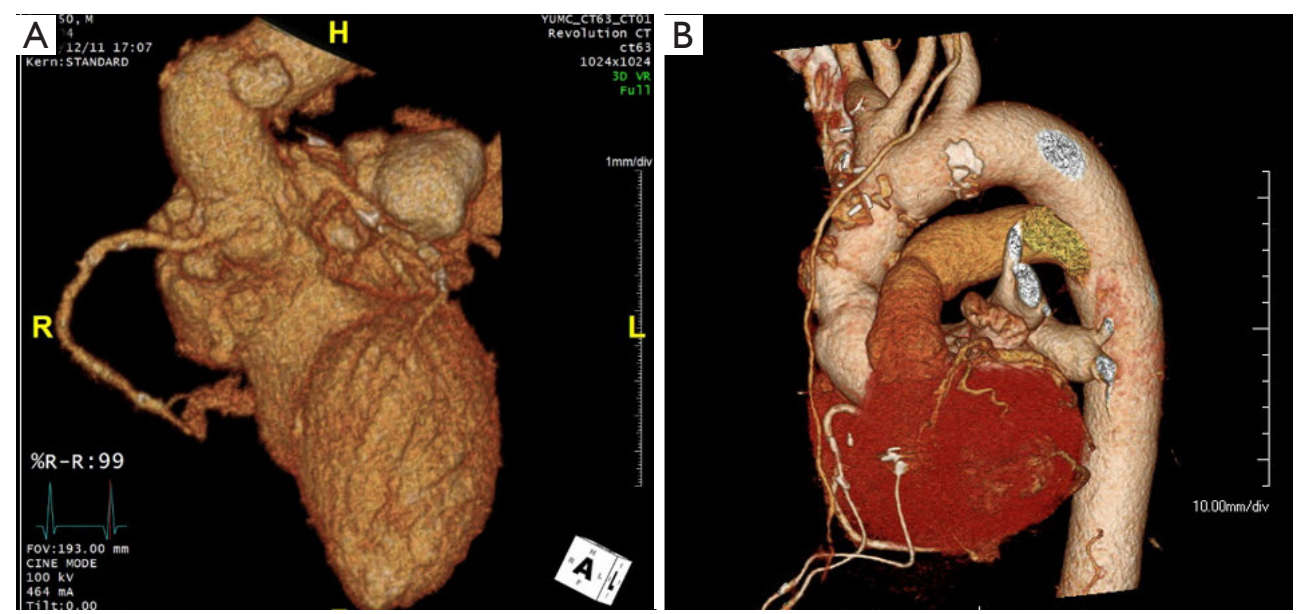

Figure 2 Cardiac computed tomography scan. (A) Before the third surgery shows multiple anastomotic pseudoaneurysms; (B) after heart transplantation.

extensive pseudoaneurysms around the coronary button and the distal graft anastomoses.

The patient underwent a third aortic root surgery using a mechanical valved conduit. Six hours after surgery, the patient underwent cardiac resuscitation and extracorporeal membrane oxygenation (ECMO) support due to postcardiotomy cardiogenic shock and was listed for a transplant.

After an 11-day wait under ECMO, orthotopic heart transplantation was performed by median sternotomy. During cardiectomy, we found an aneurysmal sac and internal thrombus in the left atrial appendage wall with a suspicious intracardiac shunt flow (panel B in Figure 1).
The aorta from the donor was anastomosed to the previous synthetic graft, which was expected to serve as the tension distributor to prevent further dilatation or dissection. The suture line, anastomosed to the pulmonary artery, was placed close to the pulmonary artery bifurcation of the donor due to the aneurysmal dilatation of the recipient (panel B in Figure 2). The donor's heart had an ischemic time of 204 minutes and a bypass time of 193 minutes.

During surgery, therapeutic plasma exchange was utilized to prevent acute rejection due to high panel reactive antibody levels. Postoperatively, immunosuppressive therapy and intravenous solumedrol were administered for 12 days, along with mycophenolate mofetil and tacrolimus. Subsequently, 
oral prednisolone $(60 \mathrm{mg} /$ day $)$ was started. At 33 days postoperatively, the patient was transferred from the intensive care unit to a general ward. He was under rehabilitation for the remaining period and finally discharged 68 days after transplantation. During the 7 -month follow-up period, aneurysmal change of anastomosis or detachment was not found. The patient remained asymptomatic, with normal C-reactive protein levels $(3.8 \mathrm{mg} / \mathrm{L})$.

\section{Discussion}

Vascular involvement in $\mathrm{BD}$ is the most severe complication and is associated with higher mortality (4). Open surgical repair is the gold standard, but it is associated with common fatal postoperative complications, such as anastomotic pseudoaneurysms or graft occlusions (5). We selected a heart transplant to overcome recurrent valve detachment and pseudoaneurysm, which had satisfactory early outcomes. However, further follow-up is required to assess long-term outcomes. To our knowledge, this is the first report of heart transplantation following repeat surgery for patients with BD. Heart transplantation may be a therapeutic option for patients with catastrophic BD.

\section{Acknowledgments}

Funding: None.

\section{Footnote}

Conflicts of Interest: All authors have completed the ICMJE uniform disclosure form (available at http://dx.doi. org/10.21037/jtd-20-1084). The authors have no conflicts of interest to declare.

Cite this article as: Kim HH, Yang YH, Lee SH, Youn YN. Heart transplantation for Behcet's disease after three aortic root replacements. J Thorac Dis 2020;12(7):3899-3901. doi: 10.21037/ jtd-20-1084
Ethical Statement: The authors are accountable for all aspects of the work in ensuring that questions related to the accuracy or integrity of any part of the work are appropriately investigated and resolved.

Open Access Statement: This is an Open Access article distributed in accordance with the Creative Commons Attribution-NonCommercial-NoDerivs 4.0 International License (CC BY-NC-ND 4.0), which permits the noncommercial replication and distribution of the article with the strict proviso that no changes or edits are made and the original work is properly cited (including links to both the formal publication through the relevant DOI and the license). See: https://creativecommons.org/licenses/by-nc-nd/4.0/.

\section{References}

1. Sakane T, Takeno M, Suzuki N, et al. Behçet's disease. N Engl J Med 1999;341:1284-91.

2. Hollander SA, Yasnovsky JR, Reinhartz O, et al. Behcet's disease and heart transplantation: a word of caution. J Heart Lung Transplant 2010;29:1306-8.

3. Poppas A, Coady M. Echocardiographic findings and cardiac surgical implications of aortitis and valvulitis in Behçet's disease. J Am Soc Echocardiogr 2009;22:1275-8.

4. Ohira S, Masuda S, Matsushita T. Nine-year experience of recurrent anastomotic pseudoaneurysms after thoracoabdominal aneurysm graft replacement in a patient with Behçet disease. Heart Lung Circ 2014;23:e210-3.

5. Liang MY, Yao JP, Zhang X, et al. Surgical technique for aortic regurgitation attributable to Behcet's disease: modified aortic valve replacement with reinforcement of the aortic wall. Eur J Cardiothorac Surg 2012;41:647-8. 